\title{
Cannabidiol zur Verbesserung der Genesung in frühen Stadien einer Schizophrenie
}

\section{Eine randomisierte, kontrollierte klinische Prüfung der adjuvanten Gabe zur individuellen antipsychotischen Behandlung}

\author{
F. M. Leweke ${ }^{1,2}$; C. Rohleder ${ }^{1,2}$; J. K. Müller'; D. Hirjak'; A. Meyer-Lindenberg ${ }^{1}$ \\ ${ }^{1}$ Klinik für Psychiatrie und Psychotherapie, Zentralinstitut für Seelische Gesundheit, Medizinische Fakultät Mannheim, \\ Universität Heidelberg, Mannheim; ${ }^{2}$ Brain and Mind Centre, The University of Sydney, Australien
}

\section{Schlüsselwörter \\ Cannabidiol, Schizophrenie, Remission, Adjuvans}

Zusammenfassung

Die Behandlung von Patienten mit schizophrenen Psychosen stellt trotz der Fortschritte der vergangenen Jahrzehnte immer noch eine große Herausforderung insbesondere im Hinblick auf die Genesung der Patienten dar. Obschon gerade die Akutsymptomatik mit den verfügbaren Antipsychotika verhältnismäßig gut zu behandeln ist, stellen deren eingeschränkte Wirksamkeit gegen Negativsymptomatik und kognitiven Einschränkungen und das Profil unerwünschter Wirkungen ein Problem im Hinblick auf die vollständige Wiederherstellung der sozialen und beruflichen Leistungsfähigkeit und Integration vieler Patienten dar. Im Rahmen des ESPRIT-Forschungsnetzwerkes führen wir daher eine klinische Prüfung von Cannabidiol als Zusatzmedikation bei post-akuten schizophrenen Psychosen in frühen Erkrankungsstadien durch. Hierbei werden die längerfristige Wirksamkeit und Verträglichkeit dieser Substanz geprüft, deren Wirkmechanismus die Modulation des Endocannabinoidsystems umfasst. Damit stellt Cannabidiol eine potentiell neue Wirkstoffklasse in der Behandlung von Psychosen dar, die die Genesung von Patienten hoffentlich zu verbessern hilft.
Keywords

Cannabidiol, schizophrenia, remission, add-on

Summary

Despite recent advances, the treatment of schizophrenic psychoses remains a considerable challenge when remission is the goal. Although acute psychotic symptoms respond quite well to current antipsychotics, their limited effects on negative symptoms and cognitive decline as well as their side-effect profile are still problematic regarding full recovery of social and vocational performance and integration for a substantial number of patients. The ESPRIT research network is focussed on early stages of psychosis and will examine the therapeutic potential of cannabidol as an add-on treatment in post-acute schizophrenia. In this double blind randomized clinical trial, longer-term efficacy and safety of the compound will be investigated. Cannabidiol acts by modulating the function of the endocannabinoid system. Thereby, it represents a potentially new class of antipsychotic compounds, hopefully effective to improve recovery of schizophrenic patients.

Enhancing recovery in early schizophrenia by randomized controlled add-on of cannabidiol to an individualized antipsychotic treatment Nervenheilkunde 2018; 37: 319-322 eingegangen am: 10. März 2018 angenommen am: 20. März 2018
Schizophrene Erkrankungen stellen häufige und gravierende psychiatrische Störungen dar, die trotz sachgerechter Behandlung oft nicht mit einer vollständigen $\mathrm{Ge}$ nesung einhergehen. Obwohl in diesem Zusammenhang erhebliche Anstrengungen unternommen und zweifellos Verbesserungen erreicht worden sind, ist die gegenwärtige Situation weiterhin unbefriedigend für Patienten, Angehörige und Behandler. Derzeit stehen verschiedene atypische Antipsychotika und als Medikamente der dritten Wahl weiterhin die typischen Antipsychotika für die Behandlung der Schizophrenie zur Verfügung, mit deren Hilfe sich insbesondere die Positivsymptomatik gut behandeln lässt. Allerdings ist deren Wirksamkeit bei der Behandlung der Negativsymptomatik und bei kognitiven Defiziten oft nicht ausreichend (1). Des Weiteren haben viele dieser Substanzen ein hohes Mass an unerwünschten Wirkungen, wie etwa extrapyramidale Symptome, Prolaktinerhöhungen, sexuelle Dysfunktionen oder die sehr in den Vordergrund gerückten metabolischen Komplikationen $(1,2)$. Die verfügbaren Antipsychotika wirken alle über dopaminerge oder serotonerge Rezeptoren. Jüngste Arzneimittelentwicklungen fokussieren sich auf neue Zielstrukturen, wie das glutamaterge, nikotinerge oder endocannabinoiderge System (3).

Eine vielversprechende Substanz ist dabei das Cannabidiol (4). Cannabidiol ist wie das psychotomimetische (halluzinogene) Cannabinoid $\Delta^{9}$-Tetrahydrocannabinol $\left(\Delta^{9}\right.$-THC) Bestandteil der Cannabis-sativaPflanze, hat aber keine psychotomimetischen oder suchterzeugenden Eigenschaf- 
ten. Im Gegenteil, sowohl in Tierversuchen als auch in Studien mit gesunden Probanden und Schizophrenie-Patienten ergaben sich deutliche Hinweise auf eine antipsychotische Wirkung $(4,5)$.

\section{Evidenz für Cannabidiol als neues, innovatives Antipsychotikum}

Nach ersten vielversprechenden individuellen Heilversuchen (6-8) wurde schließlich die erste doppelblinde, randomisierte, kontrollierte Arzneimittelstudie mit Cannabidiol durchgeführt und 2012 veröffentlicht (9). In dieser vierwöchigen Studie wurde die Wirkung von Cannabidiol (600-800 mg/Tag) mit der von Amisulprid (einem etablierten atypischen Antipsychotikum, 600-800 mg/Tag) bei insgesamt 42 Patienten mit einer akuten paranoiden Schizophrenie gemäß DSM-IV verglichen (9). Diese Studie zeigte, dass beide Wirkstoffe die Ausprägung der Psychopathologie im gleichen Maße über die Behandlungszeit signifikant reduzierten. Allerdings hatten die Patienten, die Cannabidiol erhielten, signifikant weniger Nebenwirkungen. Es wurden weder extrapyramidale Symptome noch eine Prolaktinerhöhung oder eine Gewichtszunahme beobachtet.

Interessanterweise wurde bei den mit Cannabidiol behandelten Patienten eine signifikante Erhöhung des Serumspiegels des Endocannabinoids Anandamid beobachtet, der wiederum signifikant mit einer Verbesserung der klinischen Symptomatik assoziiert war. Daher postulierten die Autoren, dass Cannabidiol den Metabolismus oder die Wiederaufnahme von Anandamid hemmt und auf diese Weise die regulatorische Wirkung des Endocannabinoidsystems auf die dopaminerge, glutamaterge und GABA-erge Neurotransmission beeinflusst (10-13).

In-vitro-Studien konnten zeigen, dass Cannabidiol ein moderater Inhibitor der Fettsäureamidhydrolase (Fatty acid amide hydrolase, FAAH) von Nagern ist $(9,12$, 14-16) und auf diese Weise den Abbau von Anandamid und verwandten Fettsäureethanolamiden (Palmitoylethanolamid und Oleoylethanolamid) hemmt. Daher wurde zunächst angenommen, dass Cannabidiol auf diese Weise seine antipsychotische Wirksamkeit entfaltet. Eine Studie berichtete jedoch, dass Cannabidiol die humane FAAH-Enzymvariante nicht inhibiert (16). Allerdings konnte in dieser Studie auch gezeigt werden, dass Cannabidiol humane intrazelluläre Transportproteine von Anandamid und verwandten Fettsäureethanolamiden, die sogenannten Fatty acid-binding proteins (FABPs), blockiert und auf diese Weise verhindert, dass Anandamid zu den FAAH-Enzymen transportiert wird, welche im endoplasmatischen Reticulum der Zellen lokalisiert sind (16). Somit scheint Cannabidiol den Abbau von Anandamid beim Menschen nicht direkt, sondern indirekt zu blockieren, was schließlich in einem Anstieg der Anandamidspiegel resultiert.

Bereits 2004 wurde postuliert, dass eine Erhöhung des Anandamidspiegels ein regulatorischer Faktor bei der akuten Schizophrenie sein kann (17). Dabei wurde angenommen, dass eine erhöhte Aktivierung der $\mathrm{CB}_{1}$-Rezeptoren im Gehirn durch Anandamid z. B. der übermäßigen dopaminergen Transmission (einem zentralen Faktor der Pathophysiologie der Schizophrenie) entgegenwirken kann. Möglicherweise moduliert Cannabidiol aber nicht nur die Dopamin- und Glutamattransmission, sondern verbessert auch die zerebrale Glukoseutilisation. Eine Erhöhung der zerebralen Glukoseutilisation könnte sowohl über Anandamid erfolgen, welches nicht nur $\mathrm{CB}_{1}$-Rezeptoren aktiviert, sondern auch als wesentlicher endogener Ligand an PPAR- $\gamma$ (Peroxisome proliferated receptor gamma) bindet, als auch über die Erhöhung des verwandten Fettsäureethanolamids und PPAR- $\alpha$-Agonisten Oleoylethanolamid und entsprechende Stoffwechselregulationsprozesse erfolgen (18).

Die antipsychotische Wirksamkeit von Cannabidiol wurde in einer kürzlich veröffentlichten Studie von McGuire et al. repliziert (19). In dieser doppelblinden, randomisierten, kontrollierten klinischen Studie wurde die Wirksamkeit einer 6-wöchigen Gabe von Cannabidiol (1000 mg/Tag) zusätzlich zur Standardtherapie bei $88 \mathrm{~Pa}$ tienten, die an Schizophrenie oder verwandten Erkrankungen (schizoaffektive, schizophreniforme oder wahnhafte Störungen) litten, untersucht (19). Es zeigte sich, dass eine zusätzliche Behandlung mit Cannabidiol im Vergleich zu Placebo signifikant die Positivsymptomatik reduzierte. Zudem wurde Cannabidiol gut vertragen. Die beobachteten Nebenwirkungen waren auf Placeboniveau.

Da über die Hälfte der an einer Schizophrenie erkrankten Patienten ihre Medikation aufgrund von unerwünschten Nebenwirkungen, wie starke Sedierung, Gewichtzunahme, Diabetes mellitus Typ 2, Hypertriglyceridämie, sexueller Dysfunktionen oder Bewegungsstörungen, zumindest zeitweilig absetzen, könnte einem Präparat wie Cannabidiol mit nur leichten Nebenwirkungen eine wichtige Bedeutung zukommen.

Aufgrund des günstigen Nebenwirkungsprofils und des potenziell neuen Wirkmechanismus ist Cannabidiol ein vielversprechender Kandidat für weitere klinische Studien. Allerdings liegen momentan noch keine Daten über die langfristige Sicherheit und Wirksamkeit vor. Diese Daten werden nun in einer 12-monatigen doppelblinden, randomisierten, kontrollierten klinischen Studie (CBDESPRIT, NCT02926859, DRKS00011151) untersucht, in der Patienten Cannabidiol (800 mg/Tag) oder Placebo in den ersten sechs Monaten zusätzlich zu ihrer individualisierten antipsychotischen Therapie erhalten.

\section{CBD-ESPRIT (Enhancing Schizophrenia Prevention and Recovery through Innovative Treatments)}

Das primäre Ziel unserer doppelblinden, randomisierten und placebokontrollierten Studie CBD-ESPRIT ist die Untersuchung der Wirksamkeit von Cannabidiol als Addon-Medikation zur antipsychotischen Monotherapie bei der Verbesserung der Remission bei Patienten im Frühstadium einer Schizophrenie.

Die sekundären Ziele dieser Studie beziehen sich auf die Sicherheit von Cannabidiol und den Einfluss auf den Metabolismus sowie die Motorik, die häufig durch Antipsychotika in Form von unerwünschten akuten extrapyramidalen Nebenwir- 
kungen und Spätdyskinesien beeinträchtigt ist. Zusätzlich wird der Zusammenhang zwischen dem Schweregrad der Symptome und den Plasmakonzentrationen von Cannabidiol und/oder Endocannabinoiden untersucht, um Informationen über die Plasmalevel sowie Wirksamkeits-Wechselbeziehung zu gewinnen.

\section{Aufbau der Studie}

Im Rahmen unserer Studie werden insgesamt 180 Patienten mit Schizophrenie gemäß DSM-IV-TR eingeschlossen und einer von zwei verschiedenen Behandlungsgruppen doppelblind zufällig zugeteilt. Nach der initialen Screening-Phase von bis zu 7 Tagen erhalten die Patienten über 24 Wochen entweder Cannabidiol (400 mg zwei Mal täglich) oder Placebo zusätzlich zur offenen individualisierten antipsychotischen Monotherapie mit einem von fünf zugelassenen atypischen Antipsychotika (Amisulprid, Aripiprazol, Olanzapin, Quetiapin oder Risperidon). Die Add-on-Medikation wird dann schrittweise über zwei Wochen reduziert. Nach Beendigung der Add-on-Medikation werden die Patienten weitere 24 Wochen nachverfolgt, um zu überprüfen, ob von einer Cannabidiolbehandlung langfristig positive Effekte ausgehen können ( $\triangleright$ Abb. 1). Die gesamte Studiendauer für den einzelnen Patienten beträgt somit ein Jahr.

Die Wirksamkeit der Behandlungen, mögliche Nebenwirkungen und Sicherheitsaspekte werden während regelmäßiger Visiten während sowie nach Beendigung der Behandlungszeit mittels klinischer Interviews und Skalen, neuropsychologischer Testungen, Blutanalysen und Elektrokardiogrammen (EKG) eingeschätzt.

\section{Ein- und Ausschlusskriterien}

Eingeschlossen werden können einwilligungsfähige männliche und weibliche Patienten, im Alter zwischen 18 und 65 Jahren, mit einem Body Mass Index (BMI) zwischen 18 und $40 \mathrm{~kg} / \mathrm{m}^{2}$, die an einer schizophrenen Psychose leiden (DSM-IVTR: 295.10, 295.20, 295.30, 295.90). Die Diagnose einer Schizophrenie darf dabei vor höchstens sieben Jahren erstmals ärztlich gestellt und schriftlich dokumentiert worden sein. Die Patienten müssen eine stabile

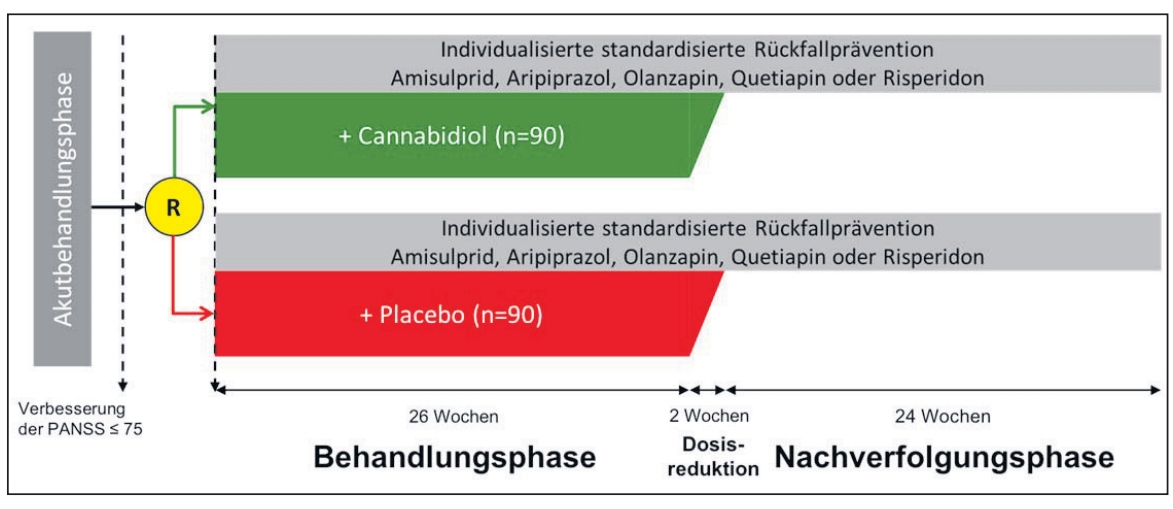

Abb. 1 Ablauf der klinischen Arzneimittelprüfung CBD-ESPRIT.

antipsychotische Standardtherapie (TAU: Treatment as usual) mit einem von fünf zugelassenen atypischen Antipsychotika (Amisulprid 50 to $1200 \mathrm{mg} / \mathrm{Tag}$, Aripiprazol 10 to $30 \mathrm{mg} / \mathrm{Tag}$, Olanzapin 5 to $20 \mathrm{mg} /$ Tag, Quetiapin 300 to $750 \mathrm{mg} / \mathrm{Tag}$, oder Risperidon 1 to $10 \mathrm{mg} / \mathrm{Tag}$ ) über mindestens 4 Wochen erhalten haben, bevor sie in die Studie eingeschlossen werden können, um sicherzustellen, dass der wesentliche Effekt der vorherigen Medikation erreicht wurde. Zur Einschätzung der Psychopathologie wird die PANSS-Skala (Positive and Negative Syndrome Scale, 20) durchgeführt, wobei die Patienten zu Beginn der Studien einen Gesamtscore von $\leq 75 \mathrm{zu}$

\section{Fazit für die Praxis}

Die Therapie von Psychosen aus dem schizophrenen Formenkreis ist weiterhin dadurch gekennzeichnet, dass nicht für alle Patienten eine zufriedenstellende Wirksamkeit und eine vollständige oder weitreichende Rückbildung der Symptomatik und Wiederherstellung der beruflichen und sozialen Leistungsfähigkeit und Integration erzielt werden kann, und die aktuellen antipsychotischen Medikamente ein relativ deutliches Profil unerwünschter Wirkungen zeigen. Daher ist die Entwicklung wirksamerer oder zumindest besser verträglicher therapeutischer Alternativen unabdingbar. Eine vielversprechende und voraussichtlich nebenwirkungsärmere Alternative kann das Cannabidiol darstellen. Basierend auf unseren Studien zur Rolle des endogenen Cannabinoidsystems bei schizophrenen Psycho- aufweisen müssen. Zudem müssen Frauen im gebärfähigen Alter eine suffiziente Verhütung während der Studie gewährleisten.

Wie in solchen Studien üblich, können Patienten mit anderen relevanten Erkrankungen der DSM-IV-Achse-I inklusive residualer Formen der Schizophrenie oder mit anderen relevanten neurologischen oder internistischen Erkrankungen nicht in die Studie eingeschlossen werden. Auch sind eine ernstzunehmende Suizidgefahr, ein positives Drogenscreening (mit Ausnahme von Cannabinoiden und Benzodiazepinen) sowie bei weiblichen Patienten eine Schwangerschaft oder Stillzeit Ausschlusskriterien.

sen untersuchen wir im Rahmen der placebokontrollierten und doppelblinden CBDESPRIT-Studie die Wirksamkeit von Cannabidiol in der Verbesserung der Remission und Rückfallverhütung bei frühen schizophrenen Psychosen (innerhalb der ersten sieben Erkrankungsjahre) nach weitgehender Rückbildung der akuten Symptome. Obwohl Cannabidiol seine Sicherheit und Wirksamkeit bei schizophrenen Psychosen und weiteren Erkrankungen erst noch unter Beweis stellen muss, ist zu hoffen, dass hieraus in der Zukunft eine medizinisch sinnvolle Ergänzung der aktuellen Wirkmechanismen und Behandlungsoptionen entwickelt werden kann. Eine breite Anwendung von Cannabidiol zur Behandlung von Psychosen außerhalb von kontrollierten klinischen Studien ist auf der Basis der vorliegenden wenigen Daten allerdings nicht zu empfehlen. 


\section{Arzneimittelrechtliche und ethische Aspekte}

Trotz der wenigen Studien, die auf eine Wirksamkeit von Cannabidiol hinweisen, soll an dieser Stelle noch einmal auf die Notwendigkeit kontrollierter Studien hingewiesen werden. Obschon durch die jüngsten gesetzgeberischen Aktivitäten auch „medizinisches Marihuana“ mit hohem Cannabidiolanteil verordnet werden kann, sind auch sehr geringe Anteile anderer Cannabinoide und insbesondere des $\Delta^{9}$-THC von Bedeutung, da dieses - im Gegensatz zu Cannabidiol - in der Leber $\mathrm{zu}$ aktiven Metaboliten verstoffwechselt wird und daher auch in geringen Mengen schon eine Wirkung entfalten kann, die den postulierten antipsychotischen Effekten des Cannabidiol entgegenstehen kann (21). Für die Zulassung von Fertigarzneimitteln zur Behandlung schizophrener Psychosen werden von den entsprechenden Behörden wie in Deutschland dem Bundesinstitut für Arzneimittel und Medizinprodukte (BfArM) umfangreiche klinische Studien erwartet. Die hier zu untersuchende Zahl von Patienten wird in den Phase-II-Studien auch nicht ansatzweise erreicht. Zudem stellt die von uns durchgeführte CBD-ESPRIT-Studie die erste Langzeitstudie mit Cannabidiol bei schizophrenen Psychosen überhaupt dar. Ein breiter Einsatz von Cannabidiol außerhalb kontrollierter Studien kann daher im Augenblick nicht empfohlen werden. Zudem ist die Kontrolle der erhobenen Befunde durch Placebogruppen aus methodischen Gründen von besonderer Bedeutung, da nur so ausgeschlossen werden kann, dass eine ggf. nicht wirksame Substanz zu einem breiten therapeutischen Einsatz kom- men kann. Daher ist dieser Ansatz insbesondere in Add-on-Studien wie der hier vorgestellten auch aus ethischen Gründen gut zu rechtfertigen.

\section{Interessenkonflikt}

FML ist Gesellschafter der curantis UG (haftungsbeschränkt) und hat Forschungsgelder der Acerus Pharmaceuticals, Kanada erhalten. Alle anderen Autoren erklären keine Interessenkonflikte.

\section{Literatur}

1. Leweke FM, Odorfer TM, Bumb JM. Medical needs in the treatment of psychotic disorders. Handb Exp Pharmacol 2012; 212: 165-85.

2. Parsons B, Allison DB, Loebel A, Williams K, Giller E, Romano S, et al. Weight effects associated with antipsychotics: a comprehensive database analysis. Schizophr Res 2009; 110(1-3): 103-10.

3. Mueller JK, Rohleder C, Leweke FM. What is the promise of nicotinergic compounds in schizophrenia treatment? Future Medicinal Chemistry 2016; 8: 2008-12.

4. Rohleder C, Müller JK, Lange B, Leweke FM. Cannabidiol as a potential new type of an antipsychotic. A critical review of the evidence. Frontiers in Pharmacology 2016; 7(422).

5. Leweke FM, Mueller JK, Lange B, Rohleder C. Therapeutic potential of cannabinoids in psychosis. Biol Psychiatry 2016; 79(7): 604-12.

6. Zuardi AW, Crippa JA, Hallak JE, Pinto JP, Chagas $\mathrm{MH}$, Rodrigues GG, et al. Cannabidiol for the treatment of psychosis in Parkinson's disease. J Psychopharmacol 2009; 23(8): 979-83.

7. Zuardi AW, Hallak JE, Dursun SM, Morais SL, Sanches RF, Musty RE, et al. Cannabidiol monotherapy for treatment-resistant schizophrenia. J Psychopharmacol 2006; 20(5): 683-6.

8. Zuardi AW, Morais SL, Guimaraes FS, Mechoulam R. Antipsychotic effect of cannabidiol. J Clin Psychiatry 1995; 56(10): 485-6.

9. Leweke FM, Piomelli D, Pahlisch F, Muhl D, Gerth $\mathrm{CW}$, Hoyer C, et al. Cannabidiol enhances anandamide signaling and alleviates psychotic symptoms of schizophrenia. Transl Psychiatry 2012; 2: e94.
10. Ryan D, Drysdale AJ, Pertwee RG, Platt B. Interactions of cannabidiol with endocannabinoid signalling in hippocampal tissue. Eur J Neurosci 2007; 25(7): 2093-102.

11. Sagredo O, Ramos JA, Decio A, Mechoulam R, Fernandez-Ruiz J. Cannabidiol reduced the striatal atrophy caused 3-nitropropionic acid in vivo by mechanisms independent of the activation of cannabinoid, vanilloid TRPV1 and adenosine A2A receptors. Eur J Neurosci 2007; 26(4): 843-51.

12. Bisogno T, Hanus L, De Petrocellis L, Tchilibon S, Ponde DE, Brandi I, et al. Molecular targets for cannabidiol and its synthetic analogues: effect on vanilloid VR1 receptors and on the cellular uptake and enzymatic hydrolysis of anandamide. $\mathrm{Br} \mathrm{J}$ Pharmacol 2001; 134(4): 845-52.

13. Müller-Vahl KR, Emrich HM. Cannabis and schizophrenia: towards a cannabinoid hypothesis of schizophrenia. Expert Rev Neurother 2008; 8(7): 1037-48.

14. Watanabe K, Kayano Y, Matsunaga T, Yamamoto I, Yoshimura $\mathrm{H}$. Inhibition of anandamide amidase activity in mouse brain microsomes by cannabinoids. Biol Pharm Bull 1996; 19(8): 1109-11.

15. De Petrocellis L, Ligresti A, Moriello AS, Allara M, Bisogno T, Petrosino S, et al. Effects of cannabinoids and cannabinoid-enriched Cannabis extracts on TRP channels and endocannabinoid metabolic enzymes. Br J Pharmacol 2011; 163(7): 1479-94.

16. Elmes MW, Kaczocha M, Berger WT, Leung K, Ralph BP, Wang L, et al. Fatty acid-binding proteins (FABPs) are intracellular carriers for delta9-tetrahydrocannabinol (THC) and cannabidiol (CBD). J Biol Chem 2015; 290(14): 8711-21.

17. Leweke FM, Koethe D. Cannabis and psychiatric disorders: it is not only addiction. Addict Biol 2008; 13(2): 264-75.

18. O'Sullivan SE. Cannabinoids go nuclear: evidence for activation of peroxisome proliferator-activated receptors. Br J Pharmacol 2007; 152(5): 576-82.

19. McGuire P, Robson P, Cubala WJ, Vasile D, Morrison PD, Barron R, et al. Cannabidiol (CBD) as an adjunctive therapy in schizophrenia: A multicenter randomized controlled trial. Am J Psychiatry 2018; 175(3): 225-231.

20. Kay SR, Fiszbein A, Opler LA. The positive and negative syndrome scale (PANSS) for schizophrenia. Schizophr Bull 1987; 13(2): 261-76.

21. D'Souza DC, Abi-Saab WM, Madonick S, Forselius-Bielen K, Doersch A, Braley G, et al. Delta9-tetrahydrocannabinol effects in schizophrenia: implications for cognition, psychosis, and addiction. Biol Psychiatry 2005; 57(6): 594-608. 


\section{Zertifizierte Fortbildung}

\section{Fragen zum Thema „Cannabidiol zur Verbesserung der Genesung in frühen Stadien einer Schizophrenie"}

1. Cannabidiol ist ein Bestandteil der Cannabis-sativa-Pflanze. Aus Studien an gesunden Probanden und schizophrenen Patienten ergeben sich deutliche Hinweise am ehesten auf folgende Wirkung:

a) depressiogen.

b) psychotomimetisch.

c) antipsychotisch.

d) antitussiv.

e) anxiogen.

2. In der ersten doppelblinden, randomisierten, kontrollierten Studie (2012) mit Cannabidiol (600-800 mg/Tag) wurde die Wirkung von Cannabidiol mit dem folgenden atypischen Antipsychotikum verglichen:

a) Risperidon.

b) Olanzapin.

c) Amisulprid.

d) Quetiapin.

e) Aripiprazol.

3. In der ersten doppelblinden, randomisierten, kontrollierten Studie (2012) mit Cannabidiol (600-800 mg/Tag) wurde die Wirkung von Cannabidiol an $42 \mathrm{~Pa}$ tienten mit der folgenden Erkrankung gemäß DSM-IV untersucht:

a) unipolare Depression.

b) schizoaffektive Störung.

c) bipolare affektive Störung.

d) paranoide Schizophrenie.

e) Autismus-Spektrum-Störung.

4. Die Dauer der ersten doppelblinden, randomisierten, kontrollierten Studie (2012) mit Cannabidiol (600-800 mg/ Tag) betrug:

a) 2 Wochen.

b) 4 Wochen.

c) 6 Wochen.

d) 8 Wochen.

e) 10 Wochen noids beobachtet werden:
5. Die erste doppelblinde, randomisierte, kontrollierte Studie (2012) mit Cannabidiol (600-800 mg/Tag) an 42 Patienten zeigte folgendes Ergebnis:

a) Keiner der Wirkstoffe konnte die Symptomatik über die Behandlungszeit signifikant verbessern.

b) Beide Wirkstoffe konnten die Ausprägung der Psychopathologie über die Behandlungszeit signifikant reduzieren.

c) Beide Wirkstoffe haben die Ausprägung der Psychopathologie über die Behandlungszeit signifikant verschlechtert.

d) Beide Wirkstoffe haben über die Behandlungszeit relevante Nebenwirkungen verursacht.

e) Beide Wirkstoffe haben die Ausprägung der Negativsymptomatik über die Behandlungszeit signifikant verschlechtert.

6. Bei den mit Cannabidiol (600-800 mg/ Tag) behandelten Patienten im Rahmen der Studie Leweke et al. 2012 konnte eine signifikante Erhöhung des Serumspiegels des folgenden Endocannabi-

a) $\Delta^{9}$-Tetrahydrocannabinol.

b) Cannabitriol.

c) Anandamid.

d) Cannabicyclol.

e) $\Delta^{9}$-Tetrahydrocannabivarin.

7. Das Cannabidiol ist in Deutschland wie folgt arzneimittelrechtlich eingeordnet:

a) als verschreibungspflichtige Arzneisubstanz.

b) als Nahrungsergänzungsmittel.

c) als homöopathischer Wirkstoff.

d) als Betäubungsmittel.

e) als apothekenpflichtiges, nicht verschreibungspflichtiges Arzneimittel.

8. Von welchen Autoren wurde die Wirksamkeit von Cannabidiol (1000 mg/Tag) in einer kürzlich erschienenen doppelblinden, randomisierten, kontrollierten Studie (2018) untersucht?

a) Peters et al.

b) McGuire et al.

c) Kahn et al.

d) Möller et al.

e) McDonald et al.
CME-Fortbildung online

Die Teilnahme an dieser durch die Ärztekammer Nordrhein zertifizierten CME-Fortbildung ist für 12 Monate ausschließlich online möglich. Zur Anmeldung gehen Sie bitte auf https://cme.thieme.de. Dort erfahren Sie auch den genauen Teilnahmeschluss. Es ist immer nur eine Antwort pro Frage zutreffend. Als Leser der Nervenheilkunde nehmen Sie kostenlos am CME-Programm teil. Je nach CME-Fortbildung erhalten Sie bis zu vier Fortbildungspunkte. Weitere Informationen zur Anmeldung und Registrierung finden Sie unter https://cme.thieme.de. Sie erhalten bei inhaltlichen und technischen Fragen tutoriellen Support.

VNR 2760512018154652879

9. Das primäre Ziel der CBD-ESPRIT-Studie ist die Untersuchung der Wirksamkeit und Verträglichkeit von Cannabidiol als Add-on-Medikation zur antipsychotischen Monotherapie bei Patienten mit:

a) langjähriger katatoner Schizophrenie.

b) einem Frühstadium der Schizophrenie.

c) therapieresistenter Schizophrenie.

d) chronischer Depression.

e) therapieresistenter schizoaffektiver Störung.

10. Wie lange muss die Tagesdosis eines atypischen Antipsychotikums mindestens stabil erhalten sein, bevor ein $\mathrm{Pa}$ tient an der CBD-ESPRIT-Studie teilnehmen kann?

a) 2 Wochen.

b) 4 Wochen.

c) 6 Wochen.

d) 8 Wochen.

e) 10 Wochen. 